\title{
108 - THE ROLE OF ASSISTIVE TECHNOLOGIES IN PROMOTING SOCIAL HEALTH AND WELL- BEING
}

Lead: Dr. F.J.M. Meiland, Amsterdam UMC, location VUmc, department of Elderly Care Medicine. A growing number of assistive technologies are being developed and evaluated in the field of dementia. With these technologies some psychosocial interventions may be delivered with more fun and/ or efficiency, which is considered important in this field with a growing number of needs and a declining number of people that can support in these needs. Technological support systems have evolved from simple, single tools to complex and integrated systems. In an INTERDEM state of the art position paper, we have reviewed issues regarding development, usability, (cost)effectiveness, deployment and ethics of assistive technologies for community dwelling people with dementia (Meiland et al., 2017). Some of these issues will be followed up in this symposium, where different types of technologies and different research methodologies in the field of dementia are presented. Scoping review, feasibility trials and randomized controlled trials are used to study various Assistive\&Assisted Living services and computertablet interventions.

The symposium leader will introduce the topic and presentations.

In the first presentation, a feasibility study into an individual Cognitive Stimulation Therapy on a tablet will be presented by $\mathrm{H}$. Rai, followed by another feasibility study into a tablet-based intervention called FindMyApps, that will be presented by K. Beentjes. The third presenter is B. Budak and she will discuss her scoping review into Active\&Assisted Living technologies and psychosocial interventions to impact loneliness.

The three presentations:

1. The individual Cognitive Stimulation Therapy (iCST) application for people with dementia and carers: a feasibility randomized controlled trial.

Harleen Rai ${ }^{1}$, Justine Schneider ${ }^{2}$, Martin Orrell ${ }^{1}$.

${ }^{1}$ Division of Psychiatry and Applied Psychology, School of Medicine, University of Nottingham, United Kingdom

${ }^{2}$ School of Sociology and Social Policy, University of Nottingham, United Kingdom

Background: Cognitive Stimulation Therapy (CST) is a brief evidence-based psychological treatment for people with mild to moderate dementia, which has shown to improve cognition and quality of life. A touch-screen version of CST (iCST app) has been developed in collaboration with technology developers and end-users to offer CST on a novel and interactive platform. An iCST app is well placed to take together the added value of computer use and the beneficial effects of CST which may produce combined, positive effects on cognition and quality of life. This study aims to evaluate the feasibility of conducting a full-scale randomised controlled trial (RCT) with the iCST app compared to a treatment as usual (TAU) control group and, to assist the development of a protocol for a full-scale RCT.

Methods: this was a multi-centre, pragmatic, single blind, randomised two-treatment arm (iCST app vs TAU) feasibility RCT over 11 weeks. People with mild to moderate dementia and their carers were recruited in pairs. Feasibility outcomes included the acceptability of the iCST app, the recruitment-, randomization-, and retention rates, and the acceptability of the outcome measures. Outcome measures included cognition for the person with dementia, and quality of life for both the person with dementia and carer. A proportion of the sample in the experimental group was invited for a semistructured post-trial interview.

Results: 59 dyads have been recruited for the study of which 30 have been randomized to the iCST app 